\title{
Ultra-spatial synchrotron radiation for imaging molecular chemical structure: Applications in plant and animal studies
}

\author{
Peiqiang $\mathrm{Yu}^{*}$ \\ College of Agriculture and Bioresources, University of Saskatchewan, 51 Campus Drive, Saskatoon, \\ Canada, S7N $5 A 8$
}

\begin{abstract}
Synchrotron-based Fourier transform infrared microspectroscopy (S-FTIR) has been developed as a rapid, direct, non-destructive, bioanalytical technique. This technique takes advantage of synchrotron light brightness and small effective source size and is capable of exploring the molecular chemical features and make-up within microstructures of a biological tissue without destruction of inherent structures at ultra-spatial resolutions within cellular dimension. To date there has been very little application of this advanced synchrotron technique to the study of plant and animal tissues' inherent structure at a cellular or subcellular level. In this article, a novel approach was introduced to show the potential of the newly developed, advanced synchrotron-based analytical technology, which can be used to reveal molecular structural-chemical features of various plant and animal tissues.
\end{abstract}

Keywords: Synchrotron, infrared microspectroscopy, imaging, molecular chemistry, plant, animal

\section{Synchrotron and infrared microspectroscopy}

Synchrotron radiation-based FTIR microspectroscopy (S-FTIR) has been developed as a rapid, direct, non-destructive and non-invasive bioanalytical technique. This technique takes advantage of synchrotron light brightness (which is usually 100-1000 times brighter than conventional globar source and has small effective source size), is capable of exploring the molecular chemistry within microstructures of biological samples with high signal to noise ratio at ultra-spatial resolutions as fine as $3-10 \mu \mathrm{m}$ [1-13]. This technique is able to provide information relating to the quantity, composition, structure and distribution of chemical constituents and functional groups in a biological tissue and can encompass a wider spectral range so that more detailed structural information can be extracted. The technique can be used to increase the fundamental understanding of plant and animal tissues' molecular structures at the cellular level and bring a new level of understanding of analytical information [14,15]. The S-FTIR allows a very small area to be explored, providing higher accuracy and precision, allows faster data collection, reaches diffraction limit as a few $\mu \mathrm{m}$ and provides very good signal to noise ratio with highly ultraspatial resolution $[5,7-9,16]$. It can reveal plant and animal structural-chemical features within cellular dimensions $[1,10,11,17,18]$. The research also shows that synchrotron IR source does not damage any

\footnotetext{
*Address for correspondence: Peiqiang $\mathrm{Yu}, \mathrm{PhD}$, College of Agriculture and Bioresources, University of Saskatchewan, 6D34 Agriculture Building, 51 Campus Drive, Saskatoon, Canada, S7N 5A8. Tel.: +1 306966 4132; Fax: +1 3069664151 ; E-mail: peiqiang.yu@usask.ca
} 
biological tissue. To date there has been little applications of this advanced synchrotron-based analytical technique to plant and animal sciences - the study of plant and animal tissues' structure at a cellular or subcellular level. In this article, an approach was introduced to show the potential of the advanced synchrotron-based analytical technology, which can be used to reveal molecular structural-chemical features of various plant and animal tissues. Synchrotron-based FTIR microspectroscopy can be used to increase the fundamental understanding of the molecular chemical structures of plant and animal tissues within cellular dimensions.

\section{Imaging molecular chemical structure of plant tissues: applications in plant study}

Complex plant tissue contains several biological components (protein, lipid, structural and nonstructural carbohydrates, and lignin). The distribution of these biological components is very heterogeneous [10]. With ultra-spatially resolved S-FTIR, the molecular structural-chemical features of the plant tissues within cellular dimensions can be imaged. To demonstrate how the S-FTIR can be used for imaging plant molecular chemical structure to reveal plant structure characteristics, the following is a review of applications of the synchrotron-based technique.

\subsection{Imaging molecular chemistry of pioneer $39 p 78$ corn and AC Barrie wheat}

These studies [11,19] was to use the synchrotron technique (S-FTIR) to image molecular chemistry of Pioneer 39P78 corn and AC Barrie wheat in order to reveal spatial intensity and distribution of chemical functional groups in corn tissue. This experiment was performed at the U2B station of National Synchrotron Light Source in Brookhaven National Laboratory (NSLS-BNL, New York). The Pioneer corn and $\mathrm{AC}$ Barrie wheat tissue were imaged from outside to inside structure under peaks at 1736 (carbonyl $\mathrm{C}=\mathrm{O}$ ester), 1510 (aromatic compound-lignin), 1650 (amide I), 1550 (amide II), 1246 (cellulosic material), 1160 (CHO), 1150 (CHO), 1080 (CHO), 929 (CHO), 860 (CHO), 3350 (OH-NH stretching), 2929 $\left(\mathrm{CH}_{2}\right.$ stretching band) and $2885 \mathrm{~cm}^{-1}\left(\mathrm{CH}_{3}\right.$ stretching band) (e.g. Fig. 1 for AC Barrie seed molecular images). Figure 2 an example show molecular functional group ratio image, representing protein-tototal-carbohydrate-ratio in the AC Barrie wheat seed tissue. The studies showed that with synchrotron FTIR microspectroscopy, the images of molecular chemistry of plant seeds could be generated at the first time. Such information on the molecular structural-chemical features of Pioneer corn and AC Barrie wheat can also be used for structure biology study, for corn and wheat breeding program for selecting
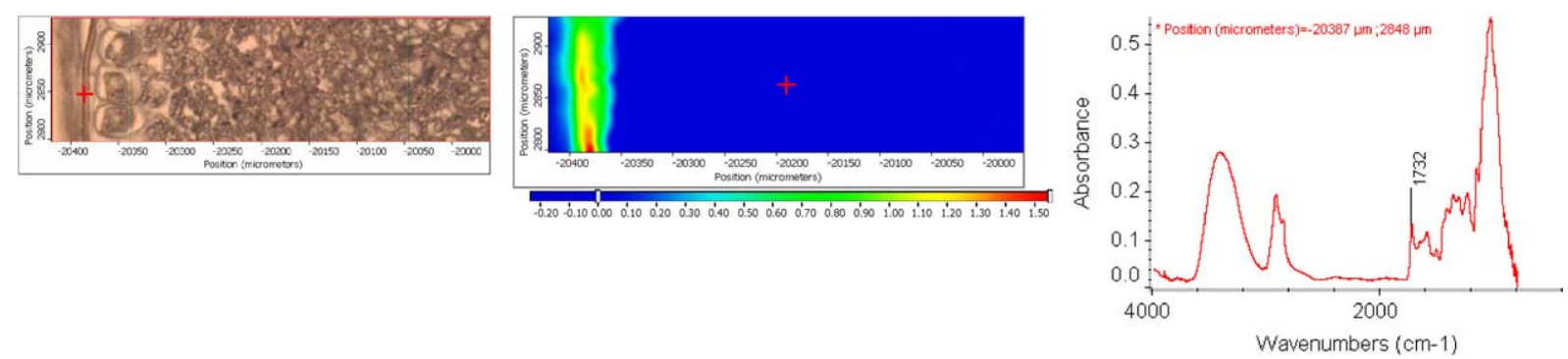

(a)

Fig. 1. Molecular functional group images of the AC Barrie wheat seed tissue from the pericarp (outside), seed coat, aleurone layer and endosperm. (1. Visible image. 2 Chemical image. 3. Spectra corresponding to the pixel at the cross-hair in the visible image.) Spectrum pixel size $10 \times 10 \mu \mathrm{m}$. (a) Area under $1732 \mathrm{~cm}^{-1}$ peak (carbonyl $\left.\mathrm{C}=\mathrm{O}\right)$. 

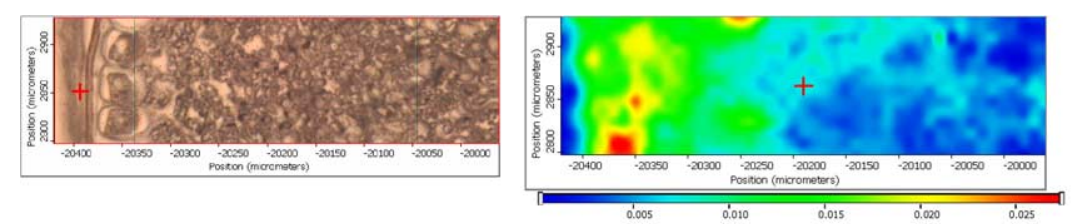

(b)
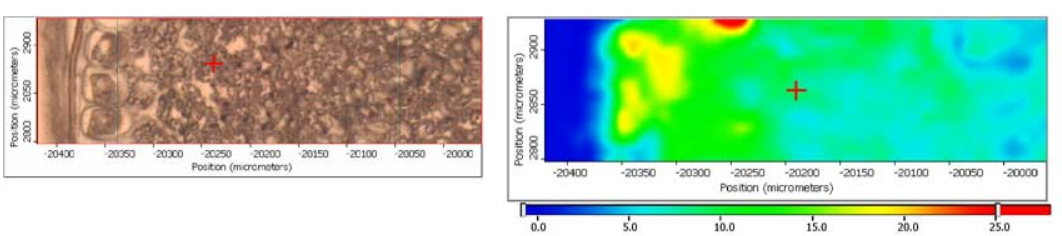

(c)
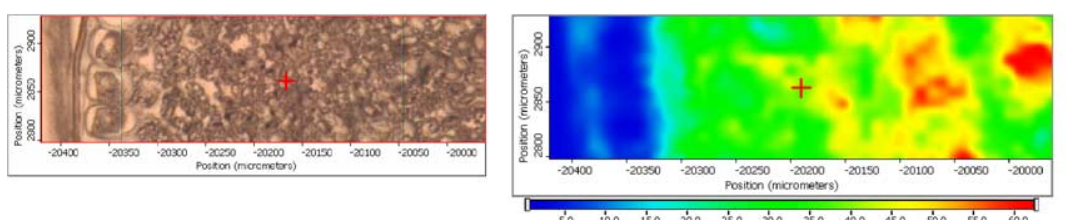

(d)
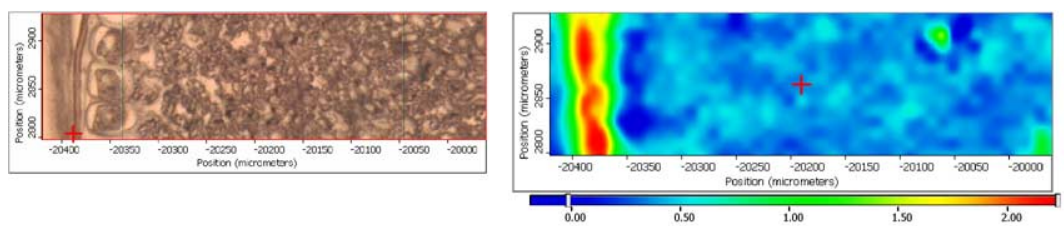

(e)
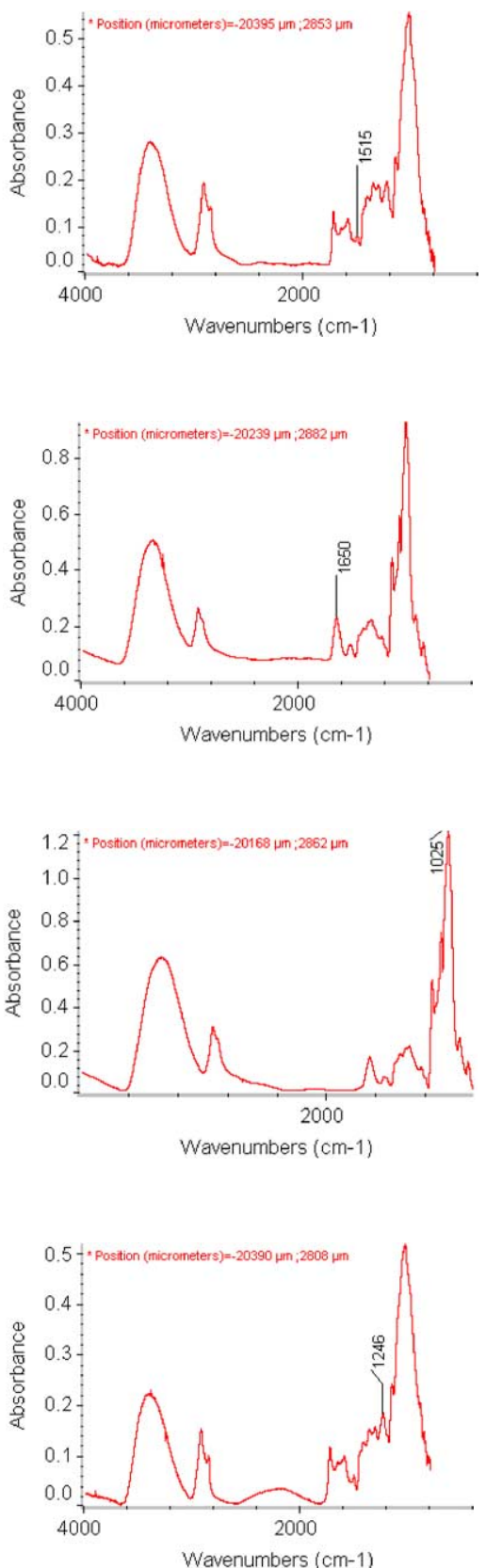

Wavenumbers $(\mathrm{cm}-1)$

Fig. 1. (b) Area under $1515 \mathrm{~cm}^{-1}$ peak (aromatic compound); (c) area under $1650 \mathrm{~cm}^{-1}$ peak (amide I); (d) area under $1025 \mathrm{~cm}^{-1}$ peak (starch); (e) area under $1246 \mathrm{~cm}^{-1}$ peak (cellulosic materials).

superior variety of corn and wheat for targeted food and feed purposes, and for prediction of corn and wheat quality and nutritive value for humans and animals. 

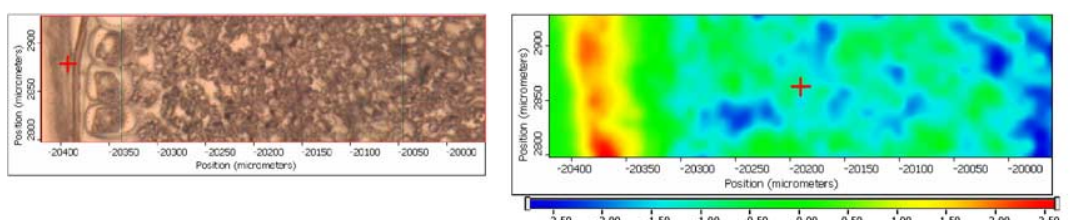

(f)
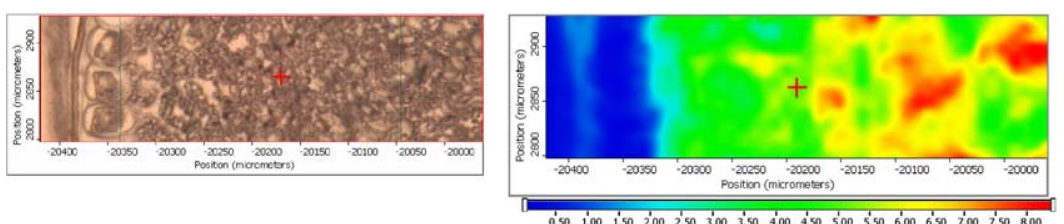

$(\mathrm{g})$
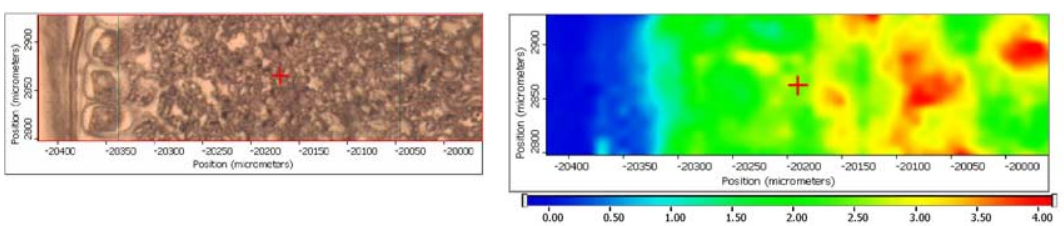

(h)
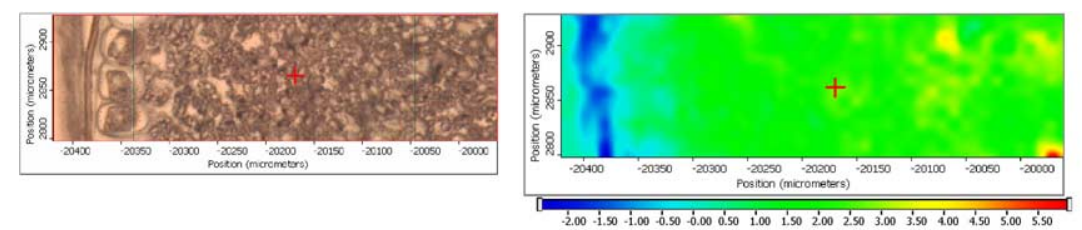

(i)
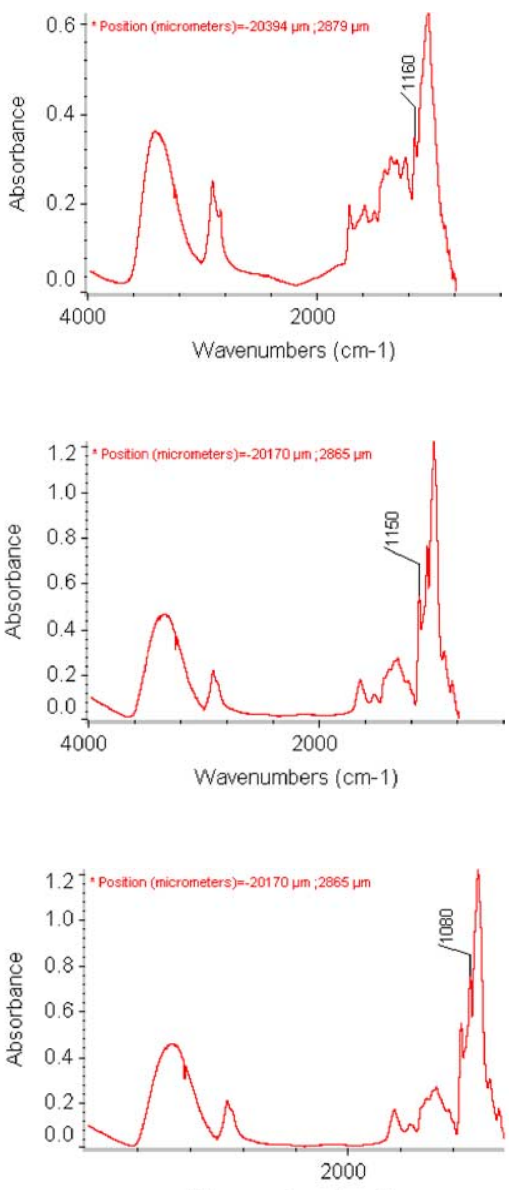

Wavenumbers $(\mathrm{cm}-1)$

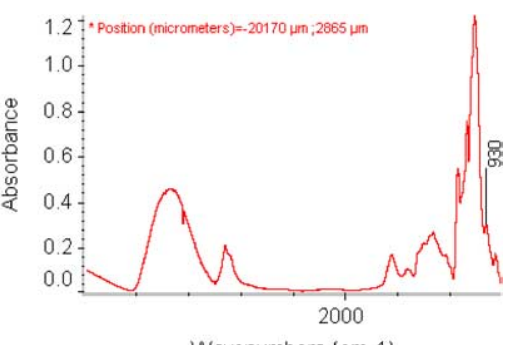

Wavenumbers $(\mathrm{cm}-1)$

Fig. 1. (f) Area under $1160 \mathrm{~cm}^{-1}$ peak (CHO); (g) area under $1150 \mathrm{~cm}^{-1}$ peak (CHO); (h) area under $1080 \mathrm{~cm}^{-1}$ peak (CHO); (i) area under $930 \mathrm{~cm}^{-1}$ peak (CHO).

\subsection{Imaging molecular chemistry of malting barley Harrington seeds}

This study [10] was to image inherent structures of barley seed tissues on a cellular level. The study showed barley intrinsic structure from pericarp, seed coat, aleurone layer and endosperm (Fig. 3). Malt- 

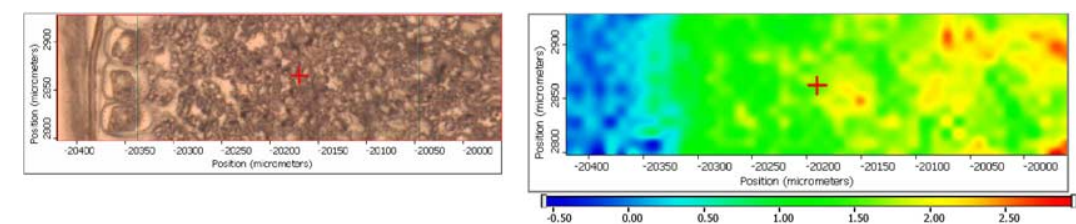

(j)
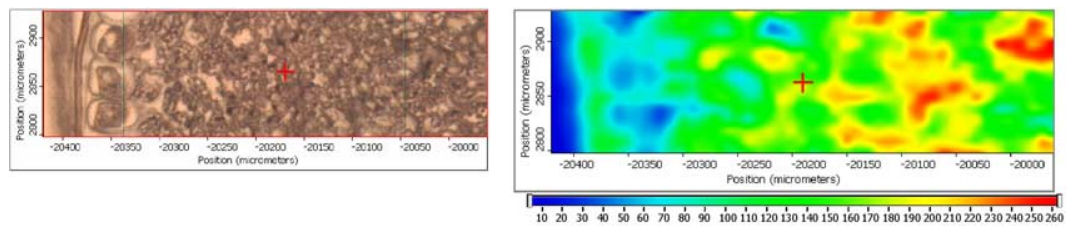

(k)
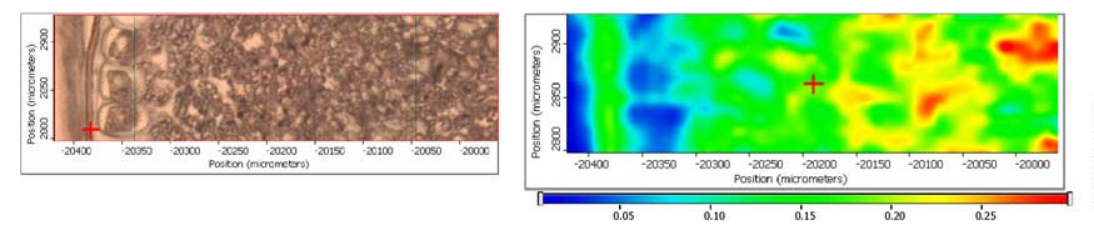

(1)
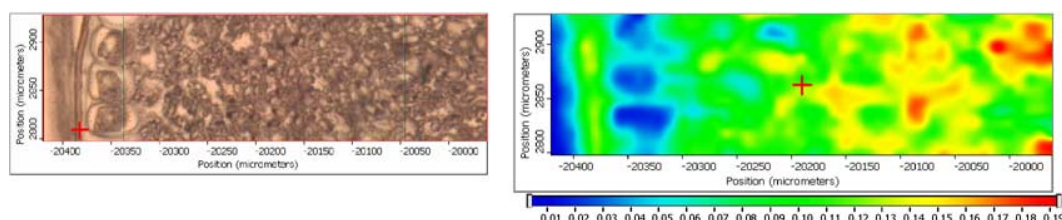

(m)

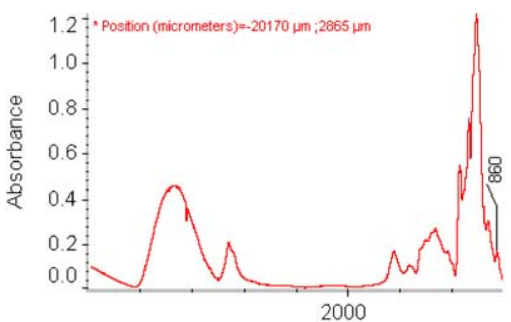

Wavenumbers $(\mathrm{cm}-1)$

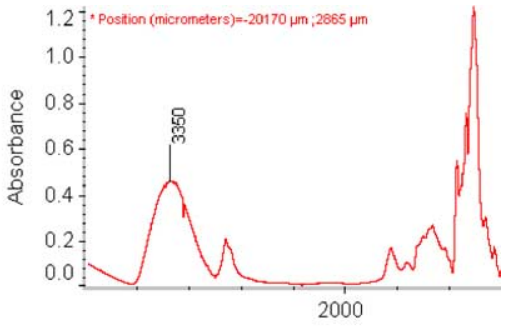

Wavenumbers $(\mathrm{cm}-1)$

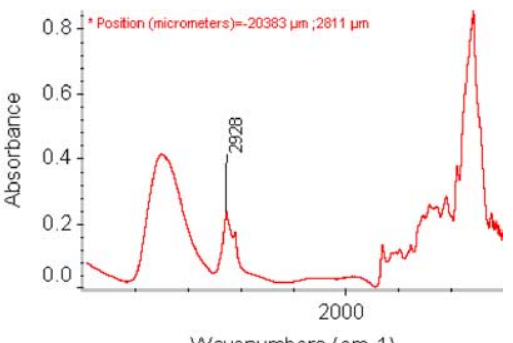

Wavenumbers $(\mathrm{cm}-1)$

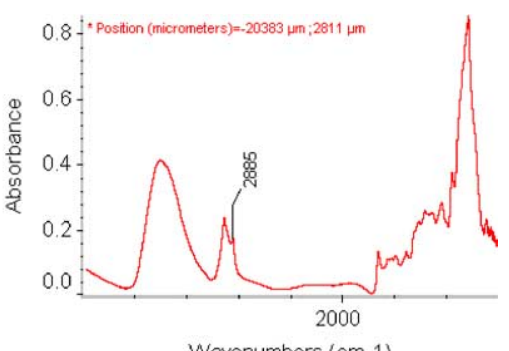

Fig. 1. (j) Area under $860 \mathrm{~cm}^{-1}$ peak (CHO); (k) area under $3350 \mathrm{~cm}^{-1}$ peak $(\mathrm{OH} \& \mathrm{NH}$ : protein and $\mathrm{CHO})$; (1) area under $2929 \mathrm{~cm}^{-1}$ peak $\left(\mathrm{CH}_{2}\right)$; (m) area under $2885 \mathrm{~cm}^{-1}$ peak $\left(\mathrm{CH}_{3}\right)$.

ing type barley has distinguished fermentation behavior [20]. Its degradation and degradation rate are very fast and high. The study showed that with the extremely bright synchrotron light, the molecular chemical spectrum with high-signal-to-noise-ratio were obtained from areas as small as $10 \times 10 \mu \mathrm{m}$ in the plant tissue, which allowed us to "see" barley tissue in a chemical sense on a cellular level [10]. 


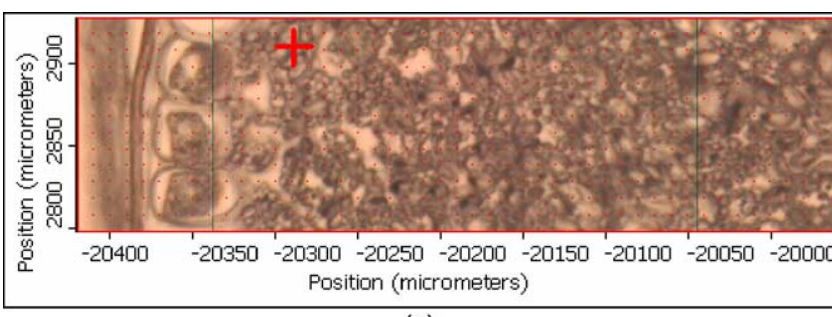

(a)

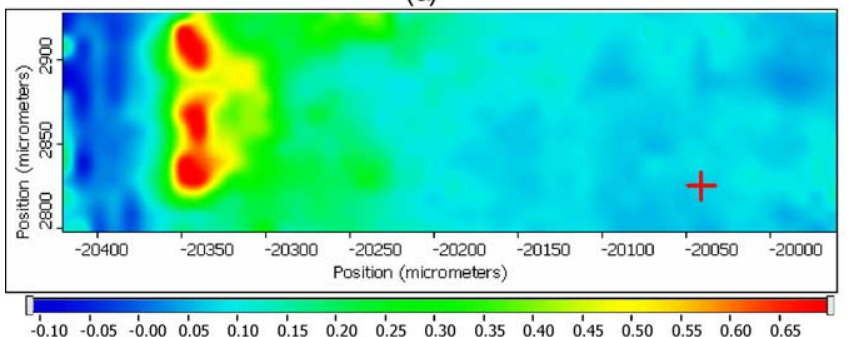

(b)

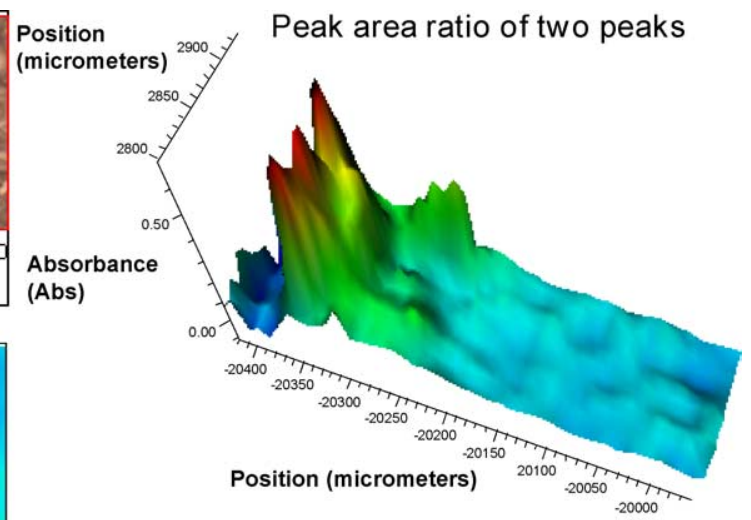

(c)

Fig. 2. Molecular functional group peak area ratio - area under the protein amide $\mathrm{I}\left(1650 \mathrm{~cm}^{-1}\right)$ bands divided by the area under the peaks between ca. 1180 and $950 \mathrm{~cm}^{-1}$ at each pixel (pixel size $10 \times 10 \mu \mathrm{m}$ ) - representing protein-to-total-carbohydrate-ratio in the AC Barrie wheat seed tissue. (a) Visible image; (b) chemical ratio image: protein to carbohydrate ratio; (c) three-D image.

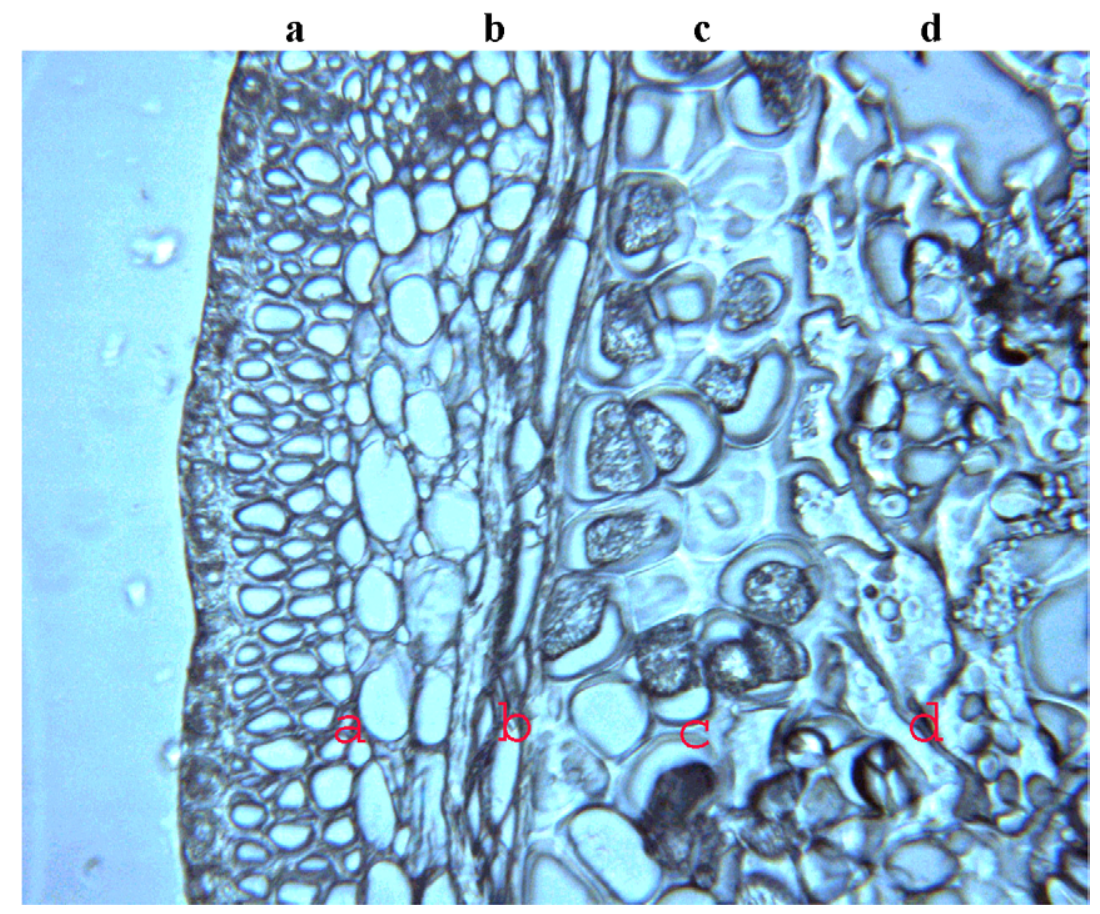

Fig. 3. Photomicrograph of cross-section of malting-type barley (cv. Harrington) showing the intrinsic structure (left to right) from the pericarp (a), seed coat (b), aleurone (c) to endosperm (d). 
The ultra-spatial resolved imaging of plant tissues by stepping in pixel sized increments was obtained. Chemical distributions of plant tissues such as lignin, cellulose, protein, lipid and total carbohydrate can be imaged across the barley tissues. These images clearly revealed the chemical information of plant intrinsic structure.

\subsection{Cluster analysis in molecular chemical imaging}

Synchrotron FTIR microspectroscopy together with cluster analysis and principal component analysis can accurately detect molecular chemical features of the micro-structure of plant tissue. The study [12] was to use a multivariate analysis - agglomerative hierarchical "cluster analysis" (CLA) [21] to more accurately analyze infrared spectra for molecular chemical imaging and reduced functional groups interference effects. The results show the CLA method gave satisfactory results and was conclusive in showing that it can discriminate and classify functional groups differences existing in different structure regions. This CLA approach for chemical imaging places synchrotron FTIR microspectroscopy at the forefront of those potential techniques that could be used in the rapid characterization of plant molecular chemical structure [12].

\section{Imaging molecular chemical structure of animal tissues: applications in animal study}

\subsection{Scrapie-infected cells, isolated prions and recombinant prion protein: a comparative study}

Kneipp et al. [22] used synchrotron FTIR microscpectroscopy to study ruminant animal disease Scrapie. Fourier-transform infrared microscopic spectra of scrapie-infected nervous tissue measured at high spatial resolution $(\sim 6 \mu \mathrm{m})$ were compared with those obtained from the purified, partly proteinase $\mathrm{K}$ digested scrapie isoform of the prion protein isolated from nervous tissue of hamsters infected with the same scrapie strain $(263 \mathrm{~K})$ to elucidate similarities/dissimilarities between prion structure investigated in situ and ex vivo. A further comparison is drawn to the recombinant Syrian hamster prion protein SHaPrP90-232 after in vitro conformational transition from the predominantly $\alpha$-helical isoform to $\beta$-sheet rich structures. It is shown that prion protein structure can be investigated within tissue and that detectability of regions with elevated $\beta$-sheet content as observed in microspectra of prion-infected tissue strongly depends on spatial resolution of the experiment.

\subsection{Characterization of prion protein structure and metal accumulation in scrapie-infected cells by synchrotron infrared and $X$-ray imaging}

Wang et al. [23] used synchrotron infrared imaging to further study ruminant animal disease. Transmissible spongiform encephalopathies (TSEs) such are neurodegenerative diseases that are characterized by the accumulation of a misfolded prion protein. During the pathogenesis, the normally a-helical prion protein (PrPC) refolds to the proteinase K-resistant abnormal form (PrPSc), which is high in b-sheet content. In this study, dorsal root ganglia from the $263 \mathrm{~K}$ hamster model of scrapie were cut into $8 \mathrm{~mm}$ thin sections and mounted onto an aluminium-coated glass slide. Single neuronal cells were mapped with synchrotron infrared microspectroscopy to study protein structure and X-ray fluorescence microprobe to determine metal accumulation. Both techniques provide a spatial resolution of 5-10 $\mu \mathrm{m}$. Results showed that some IR spectra of the infected cells exhibited a significant frequency shift of the protein amide I band, where an increased peak intensity was observed at $1631 \mathrm{~cm}^{-1}$ (attributed to $\beta$-sheet) and a decreased peak intensity at $1658 \mathrm{~cm}^{-1}$ was also seen (attributed to $\alpha$-helix) (Fig. 4). These shifts were 

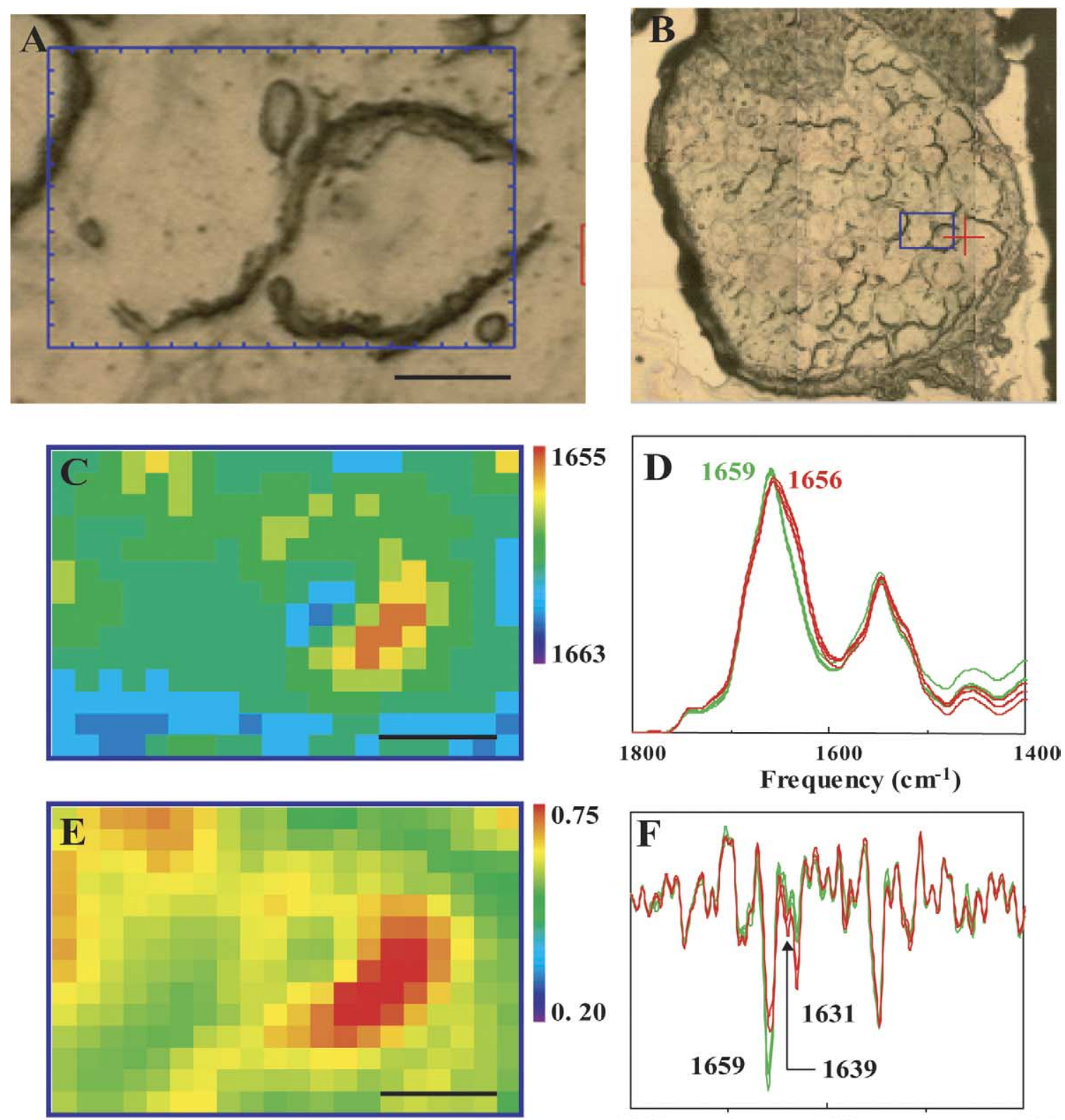

0.75

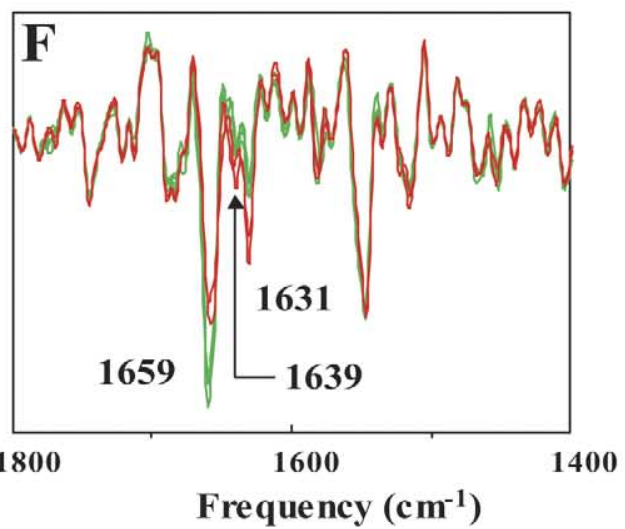

Fig. 4. (A) Visible image of two ganglion neurons from a 263K scrapie-infected hamster. (B) Visible image of a cross-section of the dorsal root ganglion from a $263 \mathrm{~K}$ scrapie-infected hamster. (C) Frequency map of the amide I band as the function of pixel location. Red and green colors indicate the two different spectral classes. (D) Absorbance spectra selected from the red and green regions of (C). (E) Peak height ratio of b-sheet/a-helix $\left(1631 / 1657 \mathrm{~cm}^{-1}\right)$ as the function of pixel location. Red and green colors indicate the two different spectral classes. (F) Second-derivative spectra selected from red and green regions of (E). The blue insets in both (A) and (B) depict the area mapped with the IR microscope. For all images, the scale bar represents $20 \mathrm{~mm}$. The IR image represents 20 pixels (horiz) $\times 14$ pixels (vert), collected with a $10 \mathrm{~mm} \times 10 \mathrm{~mm}$ aperture, a step size of $4 \mathrm{~mm}, 256 \mathrm{scans} /$ pixel, for a total data accumulation time of $560 \mathrm{~min}$ (adapted from [23]). 
not observed in non-infected cells. Adjacent sections were also stained with an antibody for the prion protein in order to confirm that the IR observations were associated with accumulated PrPSc. Finally, the same cells were measured by X-ray fluorescence microprobe, which provided the first direct comparison of PrPSc formation and metal ion accumulation. Results showed an accumulation of $\mathrm{Fe}$ in cells infected with PrPSc [23].

\subsection{Monitoring of denaturation processes in aged beef loin by Fourier transform infrared microspectroscopy}

Kirschner et al. [24] present the results of a Fourier transform infrared (FT-IR) microspectroscopic study using conventional FT-IR microscopy and FT-IR imaging to detect the denaturation process during four different heating temperatures (raw, 45,60 and $70^{\circ} \mathrm{C}$ ) spatially resolved in bovine cryosections from longissimus dorsi muscle. FT-IR imaging, employing a focal plane array detector, which allowed the simultaneous collection of spectra at 4096 pixels, enabled the investigation of the heat-induced changes in the two major meat constituents, i.e., myofibrillar and connective tissue proteins, spatially resolved. The infrared spectra of both compounds revealed that the major spectral changes involved an increase in $\beta$-sheet and a decrease in $\alpha$-helical structures, which appeared to be much more pronounced for the myofibers than for the connective tissue. These conformational changes could be correlated to the denaturation of the major meat proteins, such as myosin, actin, and collagen.

\section{Conclusions}

In conclusion, synchrotron-based technology - S-FTIR can image molecular chemical-structure features and provide functional characteristics of plant and animal tissue at high ultra-spatial resolutions. The S-FTIR images can generate spatially localized chemical information and reveal molecular chemistry information within cellular dimensions. With this available technology, we can "see" plant and animal tissues in a chemical sense. Such molecular structural chemical information can be used for plant structure biology study, for plant breeding programs for selecting superior variety of plants for targeted food and feed purposes, and for prediction of plant quality and nutritive value for humans and animals. It is believed that with the advanced synchrotron technology (S-FTIR), it will make a significant step and an important contribution to plant and animal molecular structural-chemical research.

\section{Acknowledgements}

The research has been financially supported from Natural Sciences and Engineering Research Council of Canada (NSERC, Individual Discovery Grant), Saskatchewan Agricultural Development Fund (ADF) and Canadian Light Sources (CLS) and University of Saskatchewan' synchrotron research travel fund. The National Synchrotron Light Source in Brookhaven National Laboratory (NSLS-BNL, New York, USA) is supported by the U.S. Department of Energy contract DE-AC02-98CH10886. Synchrotron Radiation Center (SRC, University of Wisconsin, Madison) is supported by the NSF under Award No. DMR-0084402. The author is grateful to John McKinnon and David Christensen (University of Saskatchewan) for their support, Nebojsa Marinkovic, Lisa Miller, Wang Qi, Alexander Ignatov, and Jennifer Bohon at U10B and U2B (NSLS-BNL, New York) and Robert Julian at Port 031 (SRC, University of Wisconsin, Madison) for helpful data collection, and Brian Rossnagel (CDC, University of 
Saskatchewan) and Vern Racz, and Philip Thacker (University of Saskatchewan) for supplying the samples.

\section{References}

[1] D.L. Wetzel, A.J. Eilert, L.N. Pietrzak, S.S. Miller and J.A. Sweat, Ultraspatially resolved synchrotron infrared microspectroscopy of plant tissue in situ, Cell. Mol. Bio. 44 (1998), 145-167.

[2] D.L. Wetzel, When molecular causes of wheat quality are known, molecular methods will supercede traditional methods, Proc. Int'l Wheat Quality Conf. II, Manhattan, Kansas, USA, May, 2001, pp. 1-20.

[3] T.K. Raab and M.C. Martin, Visualizing rhizosphere chemistry of legumes with mid-infrared synchrotron radiation, Planta 213 (2001), 881-887.

[4] D.T. Bonetta, M. Facette, T.K. Raab and C.R. Somerville, Genetic dissection of plant cell-wall biosynthesis, Biochemical Society Transactions 30 (2002), 298-301.

[5] N.S. Marinkovic, R. Huang, P. Bromberg, M. Sullivan, J. Toomey, L.M. Miller, E. Sperber, S. Moshe, K.W. Jones, E. Chouparova, S. Lappi, S. Franzen and M.R. Chance, Center for Synchrotron Biosciences' U2B beamline: An international resource for biological infrared spectroscopy, J. Synchrotron Rad. 9 (2002), 189-197.

[6] N.S. Marinkovic and M.R. Chance, Synchrotron infrared microspectroscopy, in: Encyclopedia of Molecular Cell Biology and Molecular Medicine, 2nd edn, Vol. 13, R. Meyers, ed., Wiley, New York, 2005, pp. 671-708.

[7] L.M. Miller, The impact of infrared synchrotron radiation on biology: Past, present, and future, Synchrotron Radiation News 13 (2000), 31-37.

[8] L.M. Miller, Infrared microspectroscopy and imaging. Retrieved in May from http://www.nsls.bnl.gov/newsroom/ publications/otherpubs/imaging/workshopmillerhighres.pdf, 2007.

[9] P. Dumas, Synchrotron IR microspectroscopy: A multidisciplinary analytical technique, The 6th Annual Synchrotron CLS Users' Meeting and Associated Synchrotron Workshops, University of Saskatchewan, Canada, Nov 13-15, 2003.

[10] P. Yu, J.J. McKinnon, C.R. Christensen, D.A. Christensen, N.S. Marinkovic and L.M. Miller, Chemical imaging of microstructures of plant tissues within cellular dimension using synchrotron infrared microspectroscopy, J. Agric. Food Chem. 51 (2003), 6062-6067.

[11] P. Yu, J.J. McKinnon, C.R. Christensen and D.A. Christensen, Imaging molecular chemistry of Pioneer corn, J. Agric. Food Chem. 52 (2004), 7345-7352.

[12] P. Yu, Application of cluster analysis (CLA) in feed chemical imaging to accurately reveal structural-chemical features of feeds within cellular dimension, J. Agric. Food Chem. 53 (2005), 2872-2880.

[13] L.M. Miller and P. Dumas, Chemical imaging of biological tissue with synchrotron infrared light, Biochimica et Biophysica Acta - Biomembranes 1758(7) (2006), 846-857.

[14] B.O. Budevska, Applications of vibrational spectroscopy, in: Life, Pharmaceutical and Natural Sciences, Handbook of Vibrational Spectroscopy, J.M. Chalmers and P.R. Griffiths, eds, Vol. 5, Wiley, New York, NY, USA (2002), pp. 37203732.

[15] D.L. Wetzel, P. Srivarin and J.R. Finney, Revealing protein infrared spectral detail in a heterogeneous matrix dominated by starch, Vibrational Spectroscopy 31 (2003), 109-114.

[16] H.-Y.N. Holman, K.A. Bjornstad, M.P. McNamara, M.C. Martin, W.R. McKinney and E.A. Blakely, Synchrotron infrared spectromicroscopy as a novel bioanalytical microprobe for individual living cells: Cytotoxicity considerations, J. Biomedical Optics 7 (2002), 1-10.

[17] D.L. Wetzel and S.M. LeVine, Biological applications of infrared microscpectroscopy, in: Infrared and Raman Spectroscopy of Biological Materials, H.U. Gremlich and B.Yan, eds, Marcel Dekker, New York, 2000, pp. 101-142.

[18] L.N. Pietrzak and S.S. Miller, Microchemical structure of soybean seeds revealed in situ by ultraspatially resolved synchrotron Fourier transformed infrared microspectroscopy, J. Agric. Food Chem. 53 (2005), 9304-9311.

[19] P. Yu, H. Block, Z. Niu and K. Doiron, Rapid characterization of molecular chemistry and nutrient make-up and microlocalization of internal seed tissue, J. Synchrotron Radiation (2007), in press.

[20] P. Yu, J. Meier, D.A. Christensen, B.G. Rossnagel and J.J. McKinnon, Using the NRC-2001 model and the DVE/OEB system to evaluate nutritive values of Harrington (malting-type) and Valier (feed-type) barley for ruminants, Animal Feed Science and Technology 107 (2003), 45-60.

[21] Cytospec, Software for infrared spectral imaging, V. 1.1.01, 2004.

[22] J. Kneipp, L.M. Miller, S. Spassov, F. Sokolowski, P. Lasch, M. Beekes and D. Naumann, Biopolymers 74 (2004), 163167.

[23] Q. Wang, A. Kretlow, M. Beekes, D. Naumann and L. Miller, In situ characterization of prion protein structure and metal Accumulation in scrapie-infected cells by synchrotron infrared and X-ray imaging, Vib. Spectrosc. 38 (2005), 61-69.

[24] C. Kirschner, R. Ofstad, H.J. Skarpeid, V. Host and A. Kohler, Monitoring of denaturation processes in aged beef loin by Fourier transform infrared microspectroscopy, J. Agric. Food Chem. 52 (2004), 3920-3929. 


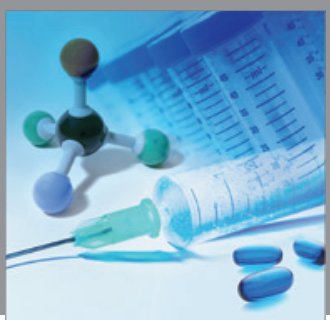

International Journal of

Medicinal Chemistry

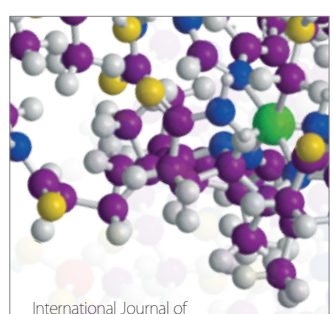

Carbohydrate Chemistry

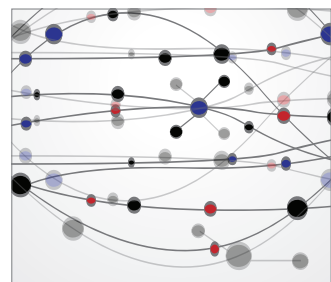

The Scientific World Journal
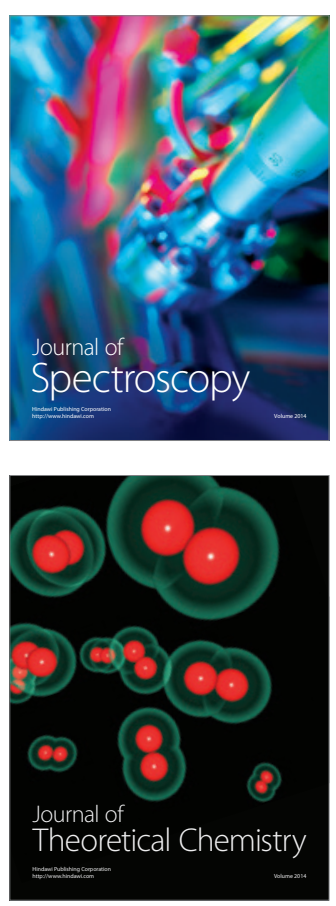
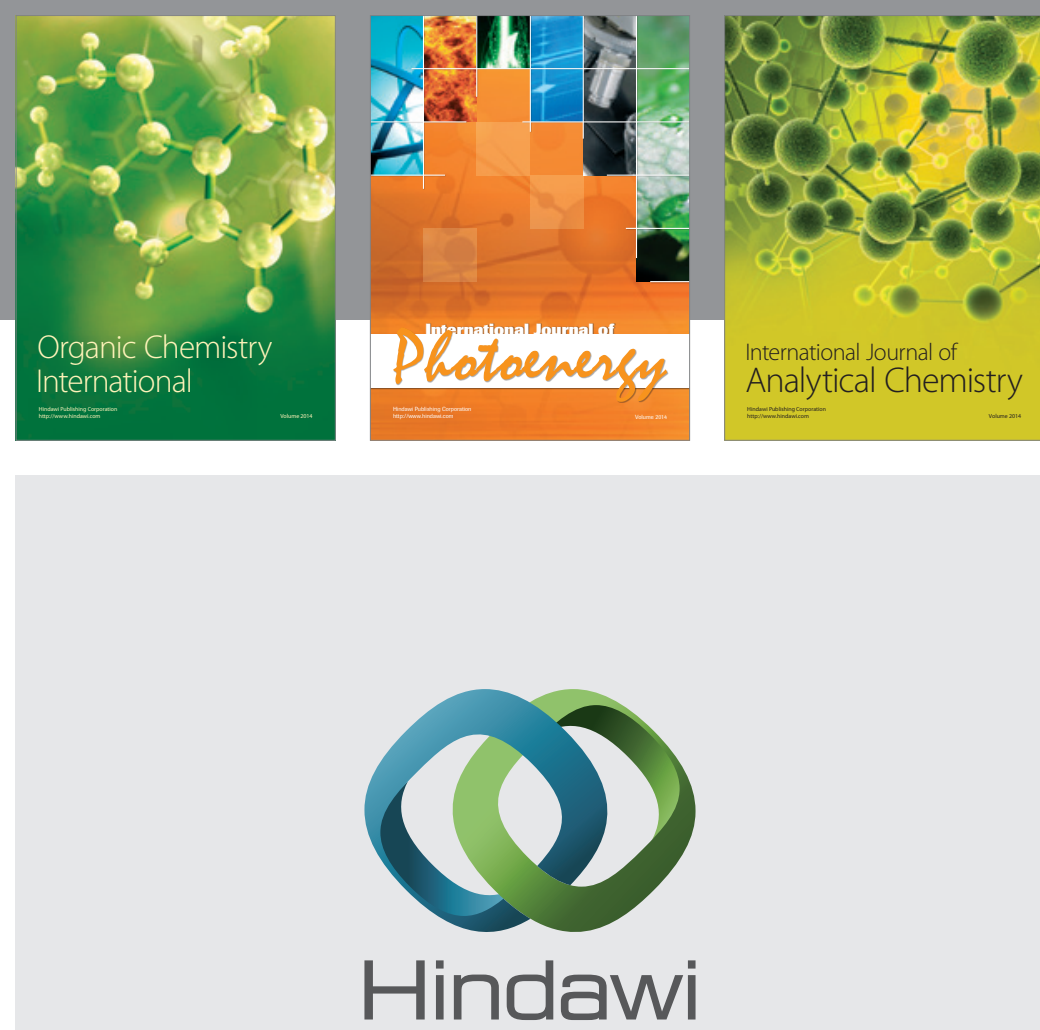

Submit your manuscripts at

http://www.hindawi.com
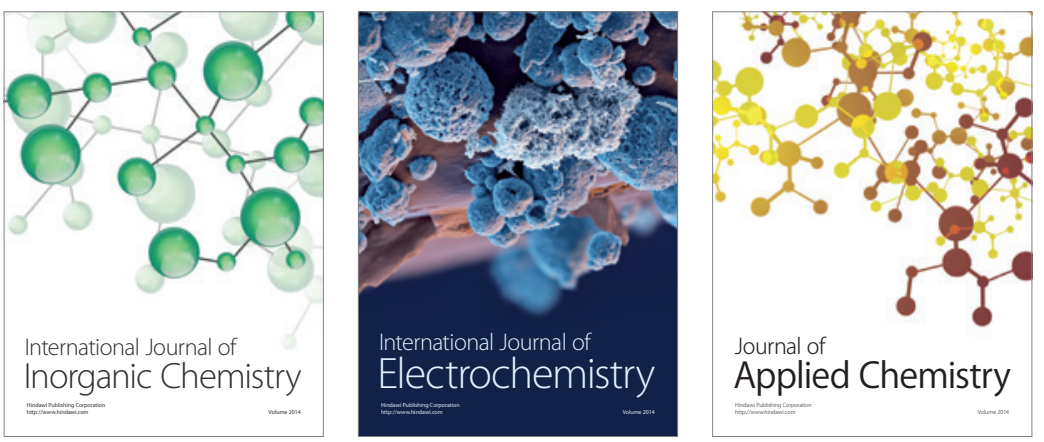

Journal of

Applied Chemistry
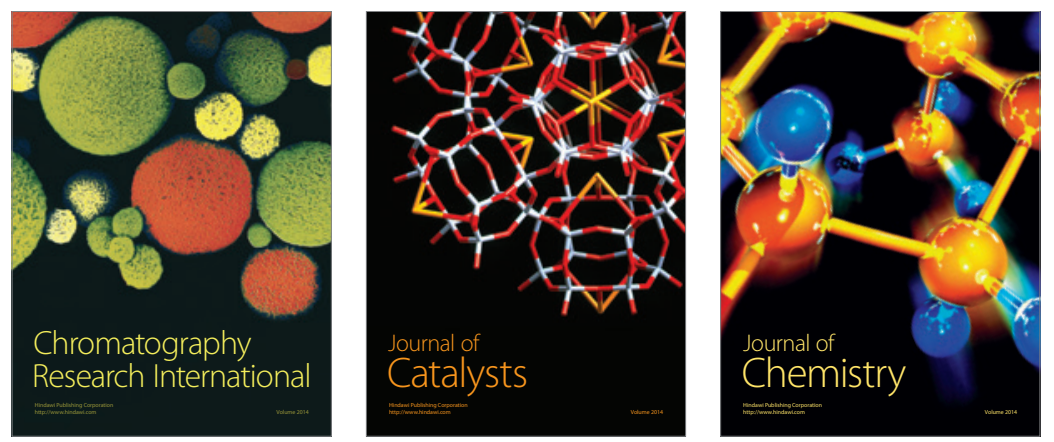
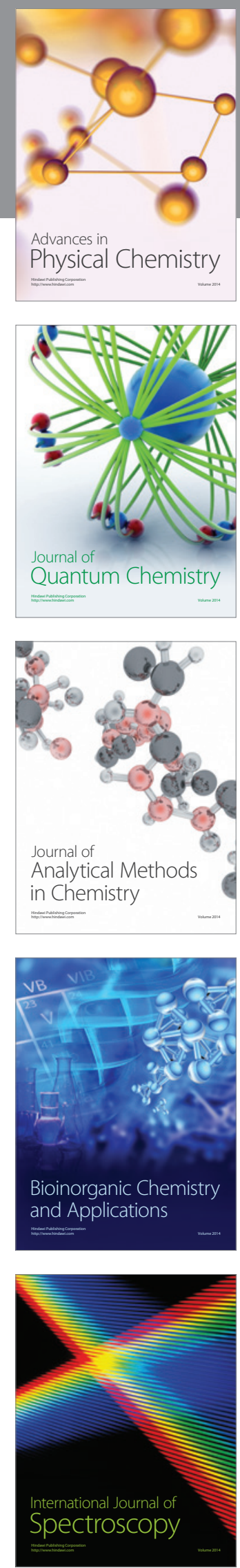